\title{
Joint Aspiration for Acute Hemarthrosis in Children Receiving Factor VIII Prophylaxis for Severe Hemophilia: 11-year Safety Data
}

\author{
Prudence J. Manners, Peter Price, Danika Buurman, Brittany Lewin, Brooke Smith, \\ and Catherine H. Cole
}

\begin{abstract}
Objective. The aims of this study were (1) to document the prevalence of acute hemarthrosis in a cohort of 46 boys with severe hemophilia A receiving full primary prophylaxis in Western Australia (WA), and (2) to investigate the safety of the WA protocol over 11 years for management of hemarthrosis.

Methods. Case review. The WA protocol involves a pediatric rheumatologist washing out all acute hemarthrosis of large joints promptly and then instilling intraarticular (IA) corticosteroids.

Results. This study showed that joint bleeds occurred in 22 boys of 46 (47.8\%). In over 11 years, 84 washouts were performed on 32 joints in 22 boys. No adverse events occurred. Fifteen of 22 boys had normal joints with a Hemophilic Joint Health Score $=0$. Fifteen boys who had had all hemarthrosis washed out had clinically normal joints $(100 \%)$. Seven boys had sustained joint damage prior to full instigation of the protocol, each having had documented hemarthrosis without aspiration. Parents needed to understand that joint bleeds constituted an emergency.

Conclusion. Of our cohort, $47.8 \%$ of patients with severe hemophilia receiving prophylaxis developed joint bleeding. The WA protocol is safe. There is evidence suggesting joint outcomes of hemophilic patients having hemarthrosis despite factor VIII prophylaxis may be much improved if there is access to a center using a procedure similar to the WA protocol. (First Release March 1 2015; J Rheumatol 2015;42:885-90; doi:10.3899/jrheum.141236)
\end{abstract}

Key Indexing Terms:
HEMOPHILIA A
ARTHROCENTESIS

FACTOR VIII

\section{HEMARTHROSIS INTRAARTICULAR STEROIDS}

\begin{abstract}
It was recognized in the 17 th century that bleeding occurs in hemophilic joints, which are progressively destroyed ${ }^{1,2}$. The quality of life of patients with hemophilia is determined by the musculoskeletal status, more than any other single factor $^{3,4}$. The advent of primary prophylaxis with replacement factor in the 1990s has vastly reduced but not eliminated the incidence of spontaneous joint bleeding and subsequent damage. Prophylaxis has been recommended since the publication by Manco-Johnson, et al in 2007 of a randomized trial of prophylaxis compared with episodic treatment, along with other similar trials ${ }^{5,6,7,8,9,10}$.
\end{abstract}

From the School of Paediatrics and Child Health, and Faculty of Medicine, University of Western Australia; Department of Haematology, Princess Margaret Hospital for Children, Subiaco, Australia.

P.J. Manners, MBBS, FRACP, School of Paediatrics and Child Health, University of Western Australia; P. Price, MBBS, FRACP, Department of Haematology, Princess Margaret Hospital for Children; D. Buurman, BSc; B. Lewin, BSC; B. Smith, BSC, Faculty of Medicine, University of Western Australia; C.H. Cole, MBBS, FRACP, FRCPA, School of Paediatrics and Child Health, University of Western Australia, and Department of Haematology, Princess Margaret Hospital for Children.

Address correspondence to Professor C.H. Cole, University of Western Australia, School of Paediatrics and Child Health, GPO Box D184,

Perth, Western Australia 6840, Australia.

E-mail: catherine.cole@health.wa.gov.au

Accepted for publication January 14, 2015.
The aims of our study were to (1) document the prevalence of acute hemarthrosis in a cohort of children in Western Australia (WA) with hemophilia A receiving primary prophylaxis, and to (2) investigate the safety over 11 years of following the WA protocol, in which a member of the pediatric rheumatology team routinely washes out and instills intraarticular (IA) corticosteroids in all acute hemarthrosis of large joints as promptly as possible.

Traditionally, joint bleeding in severe hemophilia $\mathrm{A}$ is managed conservatively with factor replacement, rest, and then physiotherapy. Our study describes the development of our center's practice of actively managing joint bleeding by removing IA blood from large joints as soon and as thoroughly as possible, followed by the instillation of IA corticosteroids.

\begin{abstract}
MATERIALS AND METHODS
A single-center retrospective review of medical records of all children with severe hemophilia A was undertaken and the operating theatre database interrogated for episodes of arthrocentesis with general anesthesia in these patients. Clinical and radiologic outcome was assessed by clinical examination, and by medical and radiology records. Our study was approved by the Child and Adolescent Health Service, Quality and Safety Committee (Quality Activity \#4473) with delegated authority from the Princess
\end{abstract}

Personal non-commercial use only. The Journal of Rheumatology Copyright @ 2015 . All rights reserved. 
Margaret Hospital for Children Human Research Ethics Committee, within which the work was undertaken.

All children with severe hemophilia A in WA were managed with factor prophylaxis. Patients were reviewed every 3 months by a hematologist, pediatric rheumatologist, pediatric physiotherapist, hemophilia nurse, and hemophilia social worker in a multidisciplinary outpatient clinic. Joint examination was performed and recorded by a pediatric rheumatologist every 3 months. From 16 years of age, patients underwent transition to adult services.

There were 46 boys with severe hemophilia A attending the clinic through this period of 11 years. Of these, 22 boys who presented with acute hemarthrosis had joints washed out. The 22 boys in the study were all boys who had their joints washed out during the 11 years. For the latter half of the time period, the policy was well entrenched, such that all acute hemarthroses were to be aspirated. Seven of the 22 boys had not had all hemarthrosis washed out, and had clearly developed joint damage prior to the full implementation of the policy. In our cohort, the majority of patients developing hemarthrosis in large joints had hemophilia A, with only 1 having hemophilia B, requiring washout of an acute hemarthrosis. We therefore chose to keep the cohort homogeneous and did not include patients with hemophilia B.

WA prophylaxis regime for hemophilic children. Prophylaxis was commenced at around 12 months of age or sooner if there had been an episode of significant bleeding. Initial treatment with recombinant factor VIII was given weekly in a dose of 50 units $/ \mathrm{kg}$. This was quickly advanced to $25-40$ units $/ \mathrm{kg}$ every second day once parents were trained to perform intravenous therapy at home.

WA protocol for management of acute hemarthrosis. Patients with suspicion of acute joint bleeding first took the usual dose factor VIII at home and were instructed not to bear weight, and to present directly to the hematology unit where the appropriate medical files were stored and medical staff were familiar with individual patients and protocols. A member of the pediatric rheumatology team was on call at all times, and acute hemarthrosis were managed according to the WA protocol. Aspiration was performed as soon as possible, ideally at presentation or within 1-3 days of the bleed. It was understood that acute inflammation of the joint occurred immediately, but the migration of monocytes to the joint heralding the chronic inflammatory cycle did not occur on the first day after the bleed, but was likely to occur by Day 4 . Hence the priority was to remove the IA blood before the chronic inflammatory cycle was initiated. However, if it had not been possible to meet this time frame, removal of blood and breakdown products from the joint still remained an important priority, thereby lessening the deposition of damaging blood products within the joint cartilage and synovium ${ }^{11}$.

A clinical history and examination were performed to confirm hemarthrosis. Other diagnoses were considered, particularly septic arthritis. Ultrasound of joints was not routinely performed. The child fasted for general anesthesia (GA). Some teenage children tolerated joint aspirations without the need for GA.

Twenty-five units $/ \mathrm{kg}$ of factor VIII were administered intravenously just prior to the procedure. Using a sterile technique, a 19- or 21-gauge needle attached to a micro-extension tube (filled with normal saline) was inserted into the joint. Image intensifier was used if necessary to confirm the correct placement of the needle. The joint was aspirated, removing as much blood as possible. Small increments of normal saline (1-10 ml, depending on joint size) were then injected through a 3-way tap attached to the micro-extension tube, followed by aspiration until aspirate was clear and colorless (Figure 1). Care was taken not to over distend the joint to avoid rupture. Following aspiration and washout, semisoluble corticosteroid was injected and washed in with a small volume of normal saline. Triamcinolone hexacetonide was used following the dose guidelines in Scott, et l $^{12}$. Using the small extension tube attached to the needle simplified the procedure because the changing of syringes for washing in and washing out did not require any manipulation or movement of the actual needle in the joint, which therefore remained correctly positioned. Any trained pediatric rheumatologist would be comfortable with this procedure, which was performed on large joints only (e.g., ankles, knees, elbows, and shoulders).

No antibiotics were required. No analgesia was required post procedure because removing blood from the joint relieved pain dramatically. Factor VIII was given the following morning to ensure no further bleeding. This may further contribute to good joint preservation. The children were discouraged from weight-bearing for $48 \mathrm{~h}$ after the procedure to allow the semisoluble steroid to remain within the joint for as long as possible and thereby to have maximum effect. In addition, rest was important after the aspiration, because IA cartilage was known to be softer and more easily damaged when it had been soaking in blood ${ }^{13}$

Where inflammatory fluid was found in the joint rather than blood, the joint was similarly aspirated, washed out, and IA corticosteroid instilled. If septic arthritis could not be excluded on aspiration, corticosteroids were withheld (Table 1).

\section{RESULTS}

Safety of the WA protocol over 11 years. No episodes of infection or extra IA bleeding occurred as a result of the procedure used on 84 occasions. No episodes of joint rupture had occurred. All children were discharged either on the same or the following day. There had been no side effects evident from the procedure.

Efficacy of WA protocol over 11 years. Forty-six boys aged $<16$ years with severe hemophilia A (factor VIII < 1\%) attended the multidisciplinary clinic regularly between 2002 and 2013. Of these, 22 boys (47.8\%) have had joint bleeds despite prophylactic factor VIII since infancy. The remainder of the boys had no recorded joint bleeds or joint problems. There were 84 joint aspirations involving 32 joints following either an emergency presentation with joint bleeding or if a new joint swelling was noted at clinic. Of 84 joints, 54 contained blood from recent bleeding and 30 contained inflammatory fluid (Table 1).

At the time of writing, 15 of the 22 boys had clinically normal joints (i.e., Hemophilic Joint Health Score $=0$ with 27 joint bleeds in total $)^{14}$. The 7 children with damaged joints had either documented joint damage prior to the instigation of the WA protocol or did not have all hemarthrosis washed out. The damaged joints included 3 left knees, 5 left ankles, and 1 right ankle.

While formal calculation of how frequently joints bled before the implementation of the protocol would be difficult to estimate, there was a sense that joints bled much less frequently once the protocol was implemented.

Three patients of particular interest. Patient 16 had a history of a high titer inhibitor to factor VIII prior to the time of the acute hemarthrosis of 1 ankle. He had commenced tolerization therapy with a plasma-derived factor VIII (Biostate) in a dose of 100 units $/ \mathrm{kg}$ daily and had received a course of rituximab at that time. He was treated with recombinant factor VIIa in a dose of $90 \mu \mathrm{g} / \mathrm{kg}$ immediately prior to joint aspirations. He had had 3 ankle aspirations. For the last 16 months, there had been no further hemarthrosis and his ankle was clinically normal.

Patient 19 has a unique phenotype such that administered 


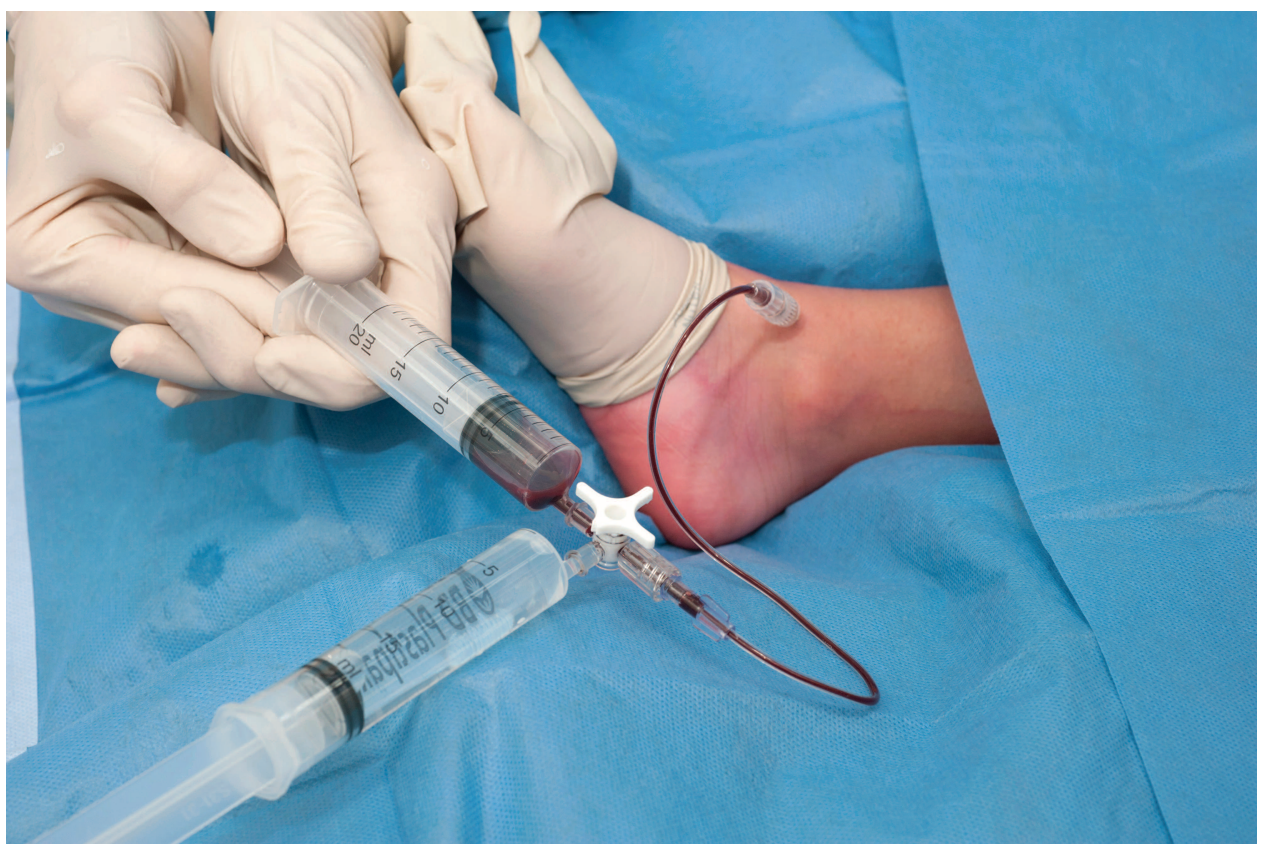

Figure 1. The Western Australia protocol of joint aspiration. Note the extension tubing and 3-way tap to avoid manipulation of the needle.

Table 1. Patients, joint injections, and nature of aspirate.

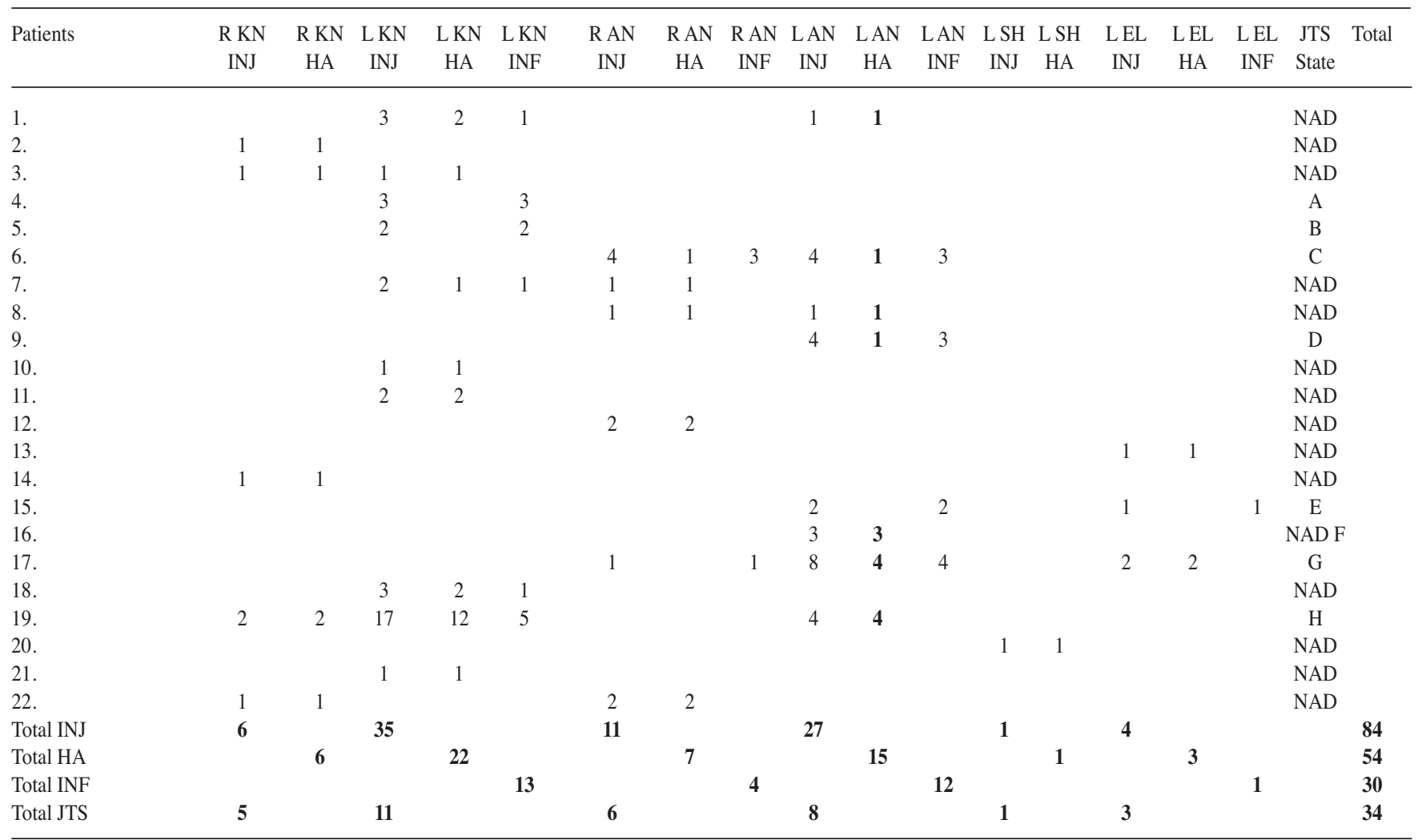

Significant data are in bold face. A: Patient with septic arthritis, not associated with injections; patient 4. B: Ruptured ACL at sport shown at MRI, otherwise no significant damage on MRI. C, D, E, and G: Four boys with significant damage to 1 or both ankles, all had documented several episodes of ankle bleeds prior to involvement in WA protocol with damage evident early and confirmed on MRI. F: Patient with inhibitor (patient 16). H: Patient with abnormal metabolism of factor VIII (patient 19). R: right; L: left; KN: knee; AN: ankle; SH: shoulder; EL: elbow; INJ: injected; HA: hemarthrosis; INF: inflammatory fluid; JTS: joints; NAD: no abnormality detected; ACL: anterior cruciate ligament; MRI: magnetic resonance imaging; WA: Western Australia. 
factor VIII has a half-life $<6 \mathrm{~h}$. The boy did not have inhibitors detected locally or in a reference laboratory, and had a normal factor VIII recovery following infusion. In recent years, he had required daily prophylactic factor infusions. By 11 years of age, his left knee had been aspirated, washed out, and injected with steroids on 17 occasions. On 12 occasions, bleeding had occurred within the joint. On 5 occasions, the aspirate was inflammatory fluid, suggesting a degree of ongoing synovitis. At 11 years of age, his knee remained functionally near normal without pain and with comparatively few magnetic resonance imaging (MRI) changes, but with some chondral damage (Figure 2). This was a significantly better outcome than would have been expected for multiple bleeds in a joint where IA blood was not removed.

Patient 4 presented at 10 months of age to the orthopedic department with a swollen, painful left knee and undiagnosed severe hemophilia A. He underwent open arthrotomy for presumed septic arthritis. An acute hemarthrosis was found and severe hemophilia A was diagnosed. Thereafter, he was managed by the WA protocol with good result, having 3 washouts with IA steroids. Two years after his third washout, he developed a painful and swollen left knee. This was initially presumed to be a hemarthrosis. At washout on the first day of symptoms, turbid fluid was found and septic arthritis was suspected and confirmed; the orthopedic

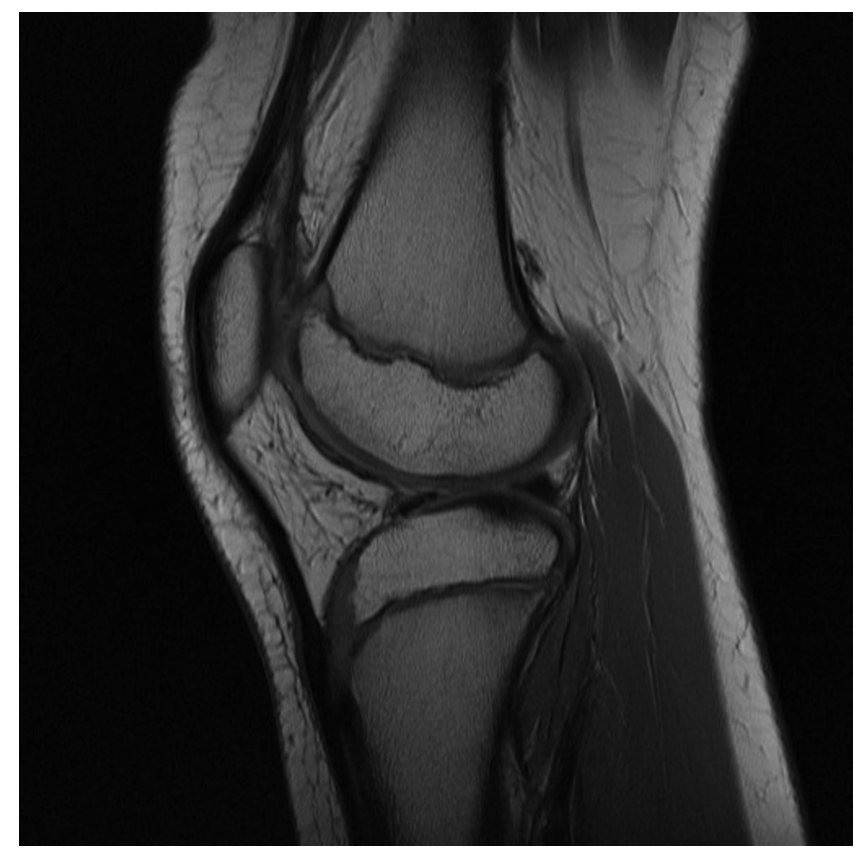

Figure 2. Patient 19: left knee aspirated, washed out, and injected with steroids on 17 occasions. On 12 occasions, bleeding had occurred within the joint. On 5 occasions, the aspirate was inflammatory fluid, suggesting a degree of ongoing synovitis. Despite this, at 11 years of age, the patient's knee remained functionally near normal without pain and with comparatively few MRI changes, but with some chondral damage. MRI: magnetic resonance imaging. surgeons performed an arthroscopic arthrotomy and washout. No steroids were instilled. IV antibiotics were given. After recovery from surgery, the knee examination was normal and fully functional. The MRI showed minimal chondral irregularity and minimal synovial thickening in the suprapatellar pouch that was the site of the orthopedic surgery during infancy (Figure 3).

\section{DISCUSSION}

In infants with severe hemophilia who do not receive prophylactic factor replacement, $44 \%$ experience their first joint bleeds in the first year of life $^{2}$ with a median age of first joint bleeding at 17 months. Ninety percent will have had spontaneous joint bleeding by 27 months. With "on demand" factor replacement therapy, $>90 \%$ of adolescents with severe hemophilia A have joint damage by 16 years with evidence of arthropathy appearing early. It is expected that by the third decade, many will have 1 severely affected joint, 2 moderately affected joints, and many deteriorating joints. Immediately upon bleeding into the joint, there is an acute inflammatory reaction manifested by an initial influx of neutrophils. Later, there is an influx of monocytes/macrophages that secrete damaging cytokines and thereby initiate the longterm cycle of inflammation and irreversible damage within the joints ${ }^{13}$. Intervening before this occurs would seem ideal. We speculate that with early intervention (aspiration and IA steroids), the acute inflammatory reaction is halted and the establishment of the longterm cycle of inflammation is prevented.

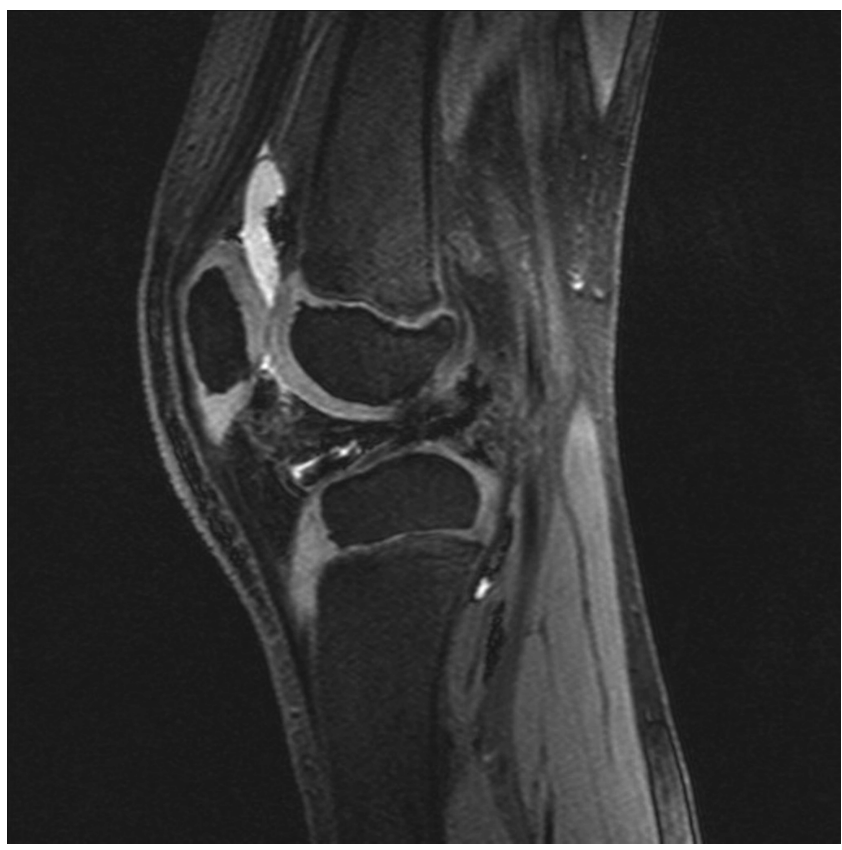

Figure 3. Hemophilia diagnosed at 10 months of age at open arthrotomy of the knee. Three subsequent episodes of bleeding managed by the Western Australia protocol and 1 episode of septic arthritis. Joint remains near normal.

Personal non-commercial use only. The Journal of Rheumatology Copyright (C) 2015. All rights reserved. 
Overall, there are abundant data published to show that since the adoption of prophylaxis, joint bleeds, though much less frequent, still occur, leading to joint damage in a significant number of hemophilic children. The WA protocol for acute hemarthrosis is aimed at maintaining normal joints for this group of children.

Our data show that nearly $50 \%$ of children treated with full prophylaxis develop joint bleeds at some time. This is higher than generally reported. This may partly be because of reporting bias in that hemarthrosis may be missed if parents are not encouraged to be acutely aware of joint bleeds. Additionally, it may be that the hemophilia community in general are not aware that there remains a significant possibility of joint bleeds despite prophylaxis. In our clinic, there is a large emphasis on joint status along with regular involvement of pediatric rheumatology at each clinic visit. The families are specifically trained to attend the hospital immediately whenever there is a possible joint bleed. The high percentage of affected boys may also reflect the Australian way of sport and outdoor activities.

While there is little reported experience on the use of IA corticosteroids in hemophilic joints, it is routine therapy in childhood arthritis and adult arthropathies of many kinds. Lessons learned from the management of these conditions inform the use of steroids in hemophilic joints. Possible side effects of IA corticosteroids documented for juvenile idiopathic arthritis are periarticular calcification, avascular necrosis of bone, Cushingoid syndrome, septic arthritis, and a small area of skin atrophy at the site of injection that disappears over 1-2 years. In the course of 11 years at our center, there were no side effects from IA steroid injections in hemophilic children.

Crystal-induced arthritis may occur 6-36 h post injection. It occurs in $3 \%$ of joint injections, is caused by temporary crystallization of injected steroid in the joint, and is painful. It is of no longterm significance. One child in our cohort had this occur following an ankle injection. After $36 \mathrm{~h}$, the symptoms disappeared. The joint recovered completely from the hemarthrosis in the usual manner.

There are 3 previous publications of interest relevant to our study. In 1975, Banta, et al ${ }^{15}$ published a series of 17 hemophilic children and young adults who had aspiration of blood from acute hemarthrosis of the knee joint followed by instillation of IA soluble corticosteroids. Following this, intravenous plasma products were given to temporarily improve coagulation. The purpose of the intervention was to restore early joint function. With all patients being discharged within $3 \mathrm{~h}$ of presentation with no instances of infection and to return to full activity within $48 \mathrm{~h}$, the intervention was deemed to be very promising. In addition, the authors noted that there seemed to be less recurrence of bleeding in comparison to when hemarthrosis had not been drained. This study did not address the problem of prevention of joint damage with most patients in the study having already sustained significant joint damage. The joints involved were not washed out with normal saline; hence, a proportion of blood would have remained in each of the aspirated joints. It was in the era before the availability of recombinant factor VIII. It is of interest that the aspiration was performed before the patient was given anticoagulation therapy. While this study supports the safety of the procedure, as well as showing promising results in faster return to joint function, it is not appropriately compared with our current study, which has different aims ${ }^{15}$.

In 1994, Rodríguez-Merchán, et al published a Spanish study that involved injecting soluble methylprednisolone into 10 knee joints with chronic hemophilic arthropathy in patients 25 years old on average ${ }^{16}$. The results were reported as excellent in 4 cases, good in 3 cases, and fair in 2 cases. This study neither addressed the problem of the removal of blood and blood products from hemarthrosis, nor the pivotal matter of progressive joint damage from the deposition of blood products within the joint. While demonstrating that IA steroids may be of benefit in hemophilic arthropathy, this study is not appropriately compared with our current study where the main feature is the prompt removal of as much blood from the acute hemarthrosis as is possible to prevent joint damage.

In 2013, Rodríguez-Merchán, et al reported a controlled study of 41 young hemophilic adults (average age $21.3 \mathrm{yrs}$ ) where blood was aspirated from acute arthroses ${ }^{17}$. It was shown that there was an excellent result in the majority of patients (i.e., "resolution within $8 \mathrm{~h}$ and not requiring any further replacement therapy"). This study confirms the safety of aspiration of acute hemarthrosis. The authors indicated that the majority of young hemophilic patients had arthropathy, and presumably most of the patients in the study were in this category. There was no attempt to wash out all the blood from the joint, or to instill corticosteroids to settle the inflammatory reaction. There was no documentation of levels of joint damage or comparison with untreated hemarthrosis. This study is not appropriately compared with our current study apart except that it supports the safety of aspiration of acute hemarthrosis ${ }^{17}$.

We hypothesize more joint bleeds will be defined in hemophilic children where a pediatric rheumatologist is included in the treating team, and that unexplained joint damage thought to be a result of micro-bleeds will prove to be because of, in part, previously undiagnosed hemarthrosis. Because hemophilic joints are more vulnerable to damage from bleeds in younger patients ${ }^{18,19}$, efforts must be focused on preservation of children's joints. The quality of the long life ahead depends largely on the musculoskeletal state. In addition, the vast community and private costs of extensive orthopedic surgery for hemophilic joints (i.e., replacement and other interventions) make it mandatory that everything possible be done to preserve joints in hemophilic children.

In our cohort, 22 boys $(47.8 \%)$ with severe hemo-

Personal non-commercial use only. The Journal of Rheumatology Copyright @ 2015 . All rights reserved. 
philia A receiving standard prophylaxis developed proven hemarthrosis.

The WA protocol for management of hemarthrosis is safe. Though this is not an efficacy study, the WA protocol showed good results (i.e., preservation of normal joints). While the removal of blood from the joint is postulated as the main ingredient for joint preservation, several factors are likely to contribute, including instillation of semisoluble steroids inhibiting the early inflammation and establishment of a chronic inflammatory cycle, parents learning to treat hemarthrosis as an emergency, 3-month surveillance by a multidisciplinary team including pediatric rheumatology, and resting of joints with hemarthrosis.

A controlled multicenter efficacy study for the WA protocol is in preparation.

\section{REFERENCES}

1. Barnes C, Wong P, Ellard DR. Reduced bone density among children with severe hemophilia. Pediatrics 2004;114:e177-81.

2. Hoots WK, Rodriguez N, Boggio L, Valentino LA. Pathogenesis of haemophilic synovitis: clinical aspects. Haemophilia 2007;13 Suppl 3:4-9.

3. Barlow JH, Stapley J, Ellard DR. Living with haemophilia and von Willebrand's: a descriptive qualitative study. Patient Educ Couns 2007;68:235-42.

4. Poonnoose PM, Srivastava A. Functional assessment of arthropathy — an international perspective. Semin Hematol 2006;43 Suppl 1:27-32.

5. Manco-Johnson MJ, Abshire TC, Shapiro AD, Riske B, Hacker MR, Kilcoyne R, et al. Prophylaxis versus episodic treatment to prevent joint disease in boys with severe hemophilia. N Engl J Med 2007;357:535-44.

6. Iorio A, Marchesini M, Marcucci M, Stobart K, Chan AK. Clotting factor concentrates given to prevent bleeding and bleeding-related complications in people with hemophilia A or B. Cochrane Database Syst Rev 2011;9:CD003429.

7. Manco-Johnson MJ, Kempton CL, Reding MT, Lissitchkov T, Goranov S, Gercheva L, et al. Randomized, controlled, parallel-group trial of routine prophylaxis vs. on-demand treatment with sucrose-formulated recombinant factor VIII in adults with severe hemophilia A (SPINART). J Thromb Haemost 2013; 11:1119-27.

8. Fischer K, Steen Carlsson K, Petrini P, Holmström M, Ljung R, van den Berg HM, et al. Intermediate-dose versus high-dose prophylaxis for severe hemophilia: comparing outcome and costs since the 1970s. Blood 2013;122:1129-36.

9. Manco-Johnson M, Blanchette VS. North American prophylaxis studies for persons with severe haemophilia: background, rationale and design. Haemophilia 2003;9 Suppl 1:44-8.

10. Brown SA, Aledort LM; Round Table Group. Economic challenges in haemophilia. Haemophilia 2005;11:64-72.

11. Roosendaal G, Lafeber FP. Pathogenesis of haemophilic arthropathy. Haemophilia 2006;12 Suppl 3:117-21.

12. Scott C, Meiorin S, Filocamo G, Lanni S, Valle M, Martinoli C, et al. A reappraisal of intra-articular corticosteroid therapy in juvenile idiopathic arthritis. Clin Exp Rheumatol 2010;28:774-81.

13. Forsyth AL, Rivard GÉ, Valentino LA, Zourikian N, Hoffman M, Monahan PE, et al. Consequences of intra-articular bleeding in haemophilia: science to clinical practice and beyond. Haemophilia 2012;18 Suppl 4:112-9.

14. Feldman BM, Funk SM, Bergstrom BM, Zourikian N, Hilliard P, van der Net J, et al. Validation of a new pediatric joint scoring system from the International Hemophilia Prophylaxis Study Group: validity of the hemophilia joint health score. Arthritis Care Res 2011;63:223-30

15. Banta JV, Boone DC, Smith CF. Arthrocentesis of the knee in acute hemophilic arthropathy. West J Med 1975;122:285-8.

16. Rodríguez-Merchán EC, Villar A, Orbe A, Magallón M. [Intra-articular methylprednisolone therapy in chronic hemophilic synovitis of the knee]. [Article in Spanish] Rev Clin Esp 1994;194:480-2.

17. Rodríguez-Merchán EC, De la Corte-Rodriguez H, Jimenez-Yuste V. Joint aspiration of acute tense knee haemarthroses in adult haemophilia A patients. Thromb Res 2013;132:778-9.

18. Jansen NW, Roosendaal G, Lafeber FP. Understanding haemophilic arthropathy: an exploration of current open issues. Br J Haematol 2008;143:632-40.

19. Roosendaal G, Tekoppele JM, Vianen ME, van den Berg HM, Lafeber FP, Bijlsma JW. Articular cartilage is more susceptible to blood induced damage at young than at old age. J Rheumatol 2000;27:1740-4. 